\title{
STAGES OF BIOFILMS FORMATION BY THE LEADING PATHOGENS IN CHILDREN WITH PYELONEPHRITIS ON CONGENITAL HYDRONEPHROSIS BACKGROUND DEPENDING ON CHILD'S AGE
}

\author{
Maryna Mishyna \\ D. P. Grynyov Department of Microbiology, Virology and Immunology ${ }^{1}$ \\ mishina1969mmm@gmail.com \\ Iryna Marchenko \\ D. P. Grynyov Department of Microbiology, Virology and Immunology ${ }^{1}$ \\ irinamarchenko2016@ukr.net \\ Vyacheslav Davydenko \\ Department of Pediatric Surgery and Pediatric Anesthesiology ${ }^{I}$ \\ davslav47@gmail.com \\ Yuliya Mozgova \\ D. P. Grynyov Department of Microbiology, Virology and Immunology ${ }^{1}$ \\ yumozgova1980@gmail.com \\ Svitlana Malanchuk \\ Department of General and Clinical Immunology and Allergology ${ }^{2}$ \\ s.malanchuk@karazin.ua \\ Serhei Dukarov \\ Department of Experimental Physics ${ }^{2}$ \\ dsv@univer.kharkov.ua \\ Yuriy Mishyn \\ Department of Dermatology, Venereology and AIDS \\ mishina1969mmm@gmail.com \\ ${ }^{1}$ Kharkiv National Medical University \\ 4 Nauky ave., Kharkiv, Ukraine, 61022 \\ ${ }^{2}$ V. N. Karazin Kharkiv National University \\ 4 Svobody sq., Kharkiv, Ukraine, 61022
}

Abstract

The aim: to study the stages of primary and secondary biofilms formation by the leading pathogens in children with pyelonephritis on congenital hydronephrosis background depending on child's age.

Materials and methods. Venflons, catheters, urine were used as material for microbiological study. Identification of microorganisms was provided with MICRO-LA-TEST ${ }^{\circledR}$ ID kits. Isolates were tested for ability to form biofilms in Petri dishes with $d=40 \mathrm{~mm}$. The morphological structure of the biofilms was studied by scanning electron microscopy.

Results: The study of structural and functional features of biofilms formation by leading pathogens of in children with pyelonephritis on congenital hydronephrosis background depending on child's age revealed a number of features and patterns. In addition to the classical stages of biofilms formation as 3D structure there was found a dissemination of planktonic cells with the release of bacteria or loss of single fragments that spread throughout the body and attach to the substrate with the formation of a new or secondary biofilm. In children under 3 years it was shown that the cocci attachment to the substrate appeared faster than in gram-negative rods and had appearance of separate structures. The longest stage of primary biofilms formation in young children was the co-aggregation. Detecting an ability to colonize with the formation of a secondary biofilm in isolates established that the longest stage was re-adsorption and the shortest was re-aggregation, which lasted 2 hours 
in all detected pathogens. In middle-aged children, the duration of adhesion stage was reduced by 1-2 hours compared with it in young children.

Conclusions. Scientific data about the stages of biofilms formation by microorganisms, causative agents of pyelonephritis in children was updated. Adhesion stage of isolates from elder children with pyelonephritis on background of congenital hydronephrosis underwent faster in the formation of secondary biofilms than in primary, and it formed the possibility for chronic process and the development of recurrences. The duration of each stage in biofilms formation by causative agents of pyelonephritis in children with congenital hydronephrosis depended on the age of the child and the properties of microorganisms.

Keywords: leading causative agents, biofilms, pyelonephritis on congenital hydronephrosis background, children.

DOI: $10.21303 / 2504-5679.2021 .001621$

\section{Introduction}

The main form of existing in bacteria during pyoinflammatory diseases, including pyelonephritis, is connected with surface community and named biofilms. In the form of biofilms bacteria appear resistant to macroorganism's defences and antibacterial drugs [1]. The role of biofilms in children urogenital pathology was assessed after confirming its existence when it was established, that the adhesive form of bacteria was not sensitive to the factors of the immune system and antibacterial drugs [2].

Since the end of the last century in medical reports have become aware of the fact that bacteria are able to form film-like macrostructures on the surfaces of medical implants and catheters [3].

It is a well-known fact that bacteria are able to colonize and form biofilms on any medical device substrates. Materials range from PVC, Polyethylene, Polyurethane, etc [4]. And this is a serious problem in medical practice, as in cases of congenital hydronephrosis in children an invasive methods (retrograde ureteropyelography, antegrade pyeloureterography, transcutaneous endopyelostomy, nephrostomy drainage, external drainage of the upper urinary tract, transurethral optical ureteropyeloscopy) and materials are widely used. Formation of biofilms in the place of inflammation turns to chronic process and development of recurrences. The leading bacteria that form biofilms in children with pyelonephritis are staphylococci, streptococci, representatives of the family Enterobacteriaceae, Pseudomona saeruginosa and others [5].

The study of biofilms is currently of great research interest mainly due to the fact, that this mode of bacterial existence creates major problems in medical practice. The ability of bacteria to form biofilms considered as a factor in their pathogenicity [6].

It is established that many chronic infections, the occurrence of which is associated with the use of medical implanted equipment, take place due to the ability of bacteria to form biofilms on the surfaces of these devices [7,8].

Biofilms can be formed on various organs and tissues in humans and animals, as well as on roots and other parts of plants. Bacteria inside biofilms show much higher resistance to antibiotics and other drugs, which makes it extremely difficult to fight against infections caused by various pathogenic bacteria [9].

The formation of biofilms by pathogenic bacteria contributes to infectious kidney damage in children with congenital hydronephrosis.

The study of ecological patterns of origin and development of microbial communities (biofilms) is a key point in the further development of medical microbiology. In this regard, the search for and study of substances that can inhibit the formation of biofilms and kill bacteria inside biofilms, is an extremely important and urgent task of antimicrobial therapy [10]. A large number of laboratories and companies around the world provide research on this problem [11].

The formation of biofilm communities proved to be one of the main strategies for the surviving of bacteria in occupied ecological loci. Being in attached state, as a part of biofilms, bacteria are protected from environmental factors and action of antibacterial substances in environment and an in host body during infection [12].

Therefore, the study of the ability to form biofilms by pathogens of pyelonephritis in children with congenital hydronephrosis and determine the duration of each stage in formation of biofilms by isolates to increase the effectiveness of antimicrobial therapy is relevant today. 
The aim of the research was to study the stages of primary and secondary biofilms formation by the leading pathogens in children with pyelonephritis on congenital hydronephrosis background depending on child's age.

\section{Materials and methods}

Urine, catheters and venflons were material for microbiological examination. Samples for the study were taken and transported to the laboratory in accordance with the requirements of collecting and transport of material for microbiological laboratories, proposed by P. L. Shupyk Medical Academy of Postgraduate Education, Kyiv [13]. All pathogens were isolated from the catheters, venflons and urine of patients on admission to the hospital before antibacterial therapy. The investigation was provided from October 2018 till October 2020. The study was approved by the ethics committee of the Kharkiv National Medical University, Ukraine (minute No. 7 dated 11 of September, 2018).

Isolation and identification of microorganisms. Microbiologic analysis was performed by using of microscopic (staining of smears and examining of morphological properties of isolates in native slides), cultural (isolation of pure culture on nutritional media), and biochemical (detection of enzymatic activity in causative agents of pyelonephritis) methods [14] and by using MICRO-LA-TEST ${ }^{\circledR}$ ID kits for standard identification micromethod.

Microbial suspension with known concentration of microbial cells was prepared on Densi-La-Meter (PLIVA-Lachema a.s., Czech Republic) by McFarland schedule and device instruction.

Optical density was measured using microplate reader «Multiskan EX» (type 355), that is a photometer with replaceable filters capable to carry out standard photometric measurements.

Synchronization of periodic culture by selection (Mitchison and Vincent method). Synchronization of periodic cultures of the studied strains was performed after establishing the growth kinetics of asynchronous culture.

The mode of periodic cultivation was so that during exponential growth the cell mass doubled from two to five times:

1. $500 \mathrm{ml}$ of sterile medium in several flasks was prepared.

2. The cells in a batch culture system were incubated until the middle of the exponential phase. The culture was rapidly cooled to $0-4{ }^{\circ} \mathrm{C}$ by putting the flasks in an ice bath. The cells were harvested by cold centrifugation at $10,000 \mathrm{~g}$ for 10 minutes.

3 . The cell pellet was resuspended in $2 \mathrm{ml}$ of ice-cold buffer $(0.1 \mathrm{M}$ potassium phosphate buffer, $\mathrm{pH}$ 7.0) on a Vortex type homogenizer.

4. Suspended cells were applied on a sterile cooled ficoll density gradient.

5. Closed Eppendorfs with cells were placed in a refrigerated homogenizer and centrifuged for $15-20 \mathrm{~min}$ at $2500 \mathrm{~g}$ in the cold environment.

6. $0.5 \mathrm{ml}$ of the lightest fraction of cells from Eppendorf was made in a flask with heated growth medium.

7. The optical density was measured and determined its gradual increase, which indicated the synchronous growth of the culture.

Sterile plastic Petri dishes $40 \mathrm{~mm}$ in diameter were used to obtain the biofilms. $4 \mathrm{ml}$ of nurtitional broth was placed in each one, and a daily culture of microorganisms isolated from patients was introduced. Incubation was carried out for $12-24$ hours at $+37^{\circ} \mathrm{C}$.

After incubation, the nutrient medium was drained, the surface of the cups was washed twice with Hanks' solution $(2 \mathrm{ml})$, fixed with $10 \%$ formalin solution in buffered $(\mathrm{pH}=7.2-7.4)$ distilled water [15].

Scanning electron microscopy (SEM) (Fig. 1) (Department of Experimental Physics, Faculty of Physics, V. N. Karazin Kharkiv national university) was used to visualize the morphological structure of biofilms in high resolution.

The Tescan Vega 3 LMH scanning electron microscope (SEM) was operated in the secondary electron mode at an accelerating voltage of $30 \mathrm{kV}$. To ensure an efficient discharge, the films were covered with a thin carbon layer. 


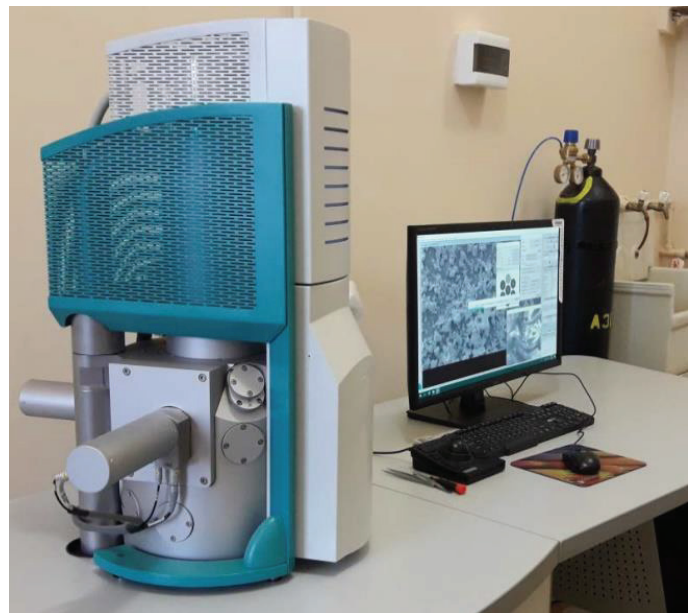

Fig. 1. Scanning electron microscope for microscopy of biofilms

\section{Results}

The study of structural and functional features of biofilms formation by the leading pathogens in children with pyelonephritis on congenital hydronephrosis background depending on child's age has revealed a number of features and patterns. In biofilms formation by isolates it was determined that the classical stages took place [16, 17]. And these stages were as the first stage - adhesion (sedimentation), the primary attachment of the pathogen to the substrate, the second stage - fixation, the final irreversible attachment of microorganism cells to the surface, the third stage - coaggregation, microcolonies with subsequent synthesis of the polymer matrix, the fourth stage - formation of clusters and mature biofilm, after the fusion of microcolonies a 3D structure is formed that can change size and shape, the fifth stage - dispersion, bacterial release or loss of single fragments that spread throughout the body and attach to substrate with the formation of a new biofilm.

At the first stage of biofilms formation by pathogens isolated from children of 0-3 years, it was found that the attachment of cocci to the substrate occurred faster than in gram-negative rods, namely: Enterococcus faecalis after 2-4 hours (Fig. 2, a), Staphylococcus epidermidis after 3-5 hours, and Escherichia coli after 6-8 hours. Also separate structures consisting of several cells with the formation of a thin film were found.

It was found that the longest stages of primary biofilms formation in young children were the co-aggregation stage (Fig. 2, b) in cocci: Enterococcus faecalis and Staphylococcus epidermidis (9 hours) and the dispersion stage lasting 12 hours in isolates of Escherichia coli.

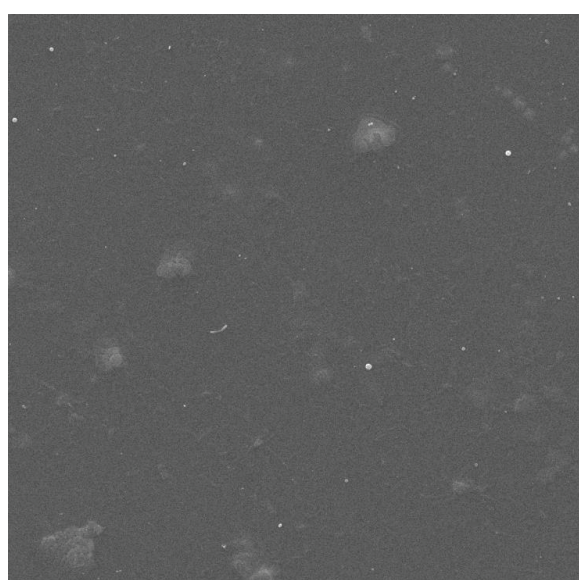

$a$

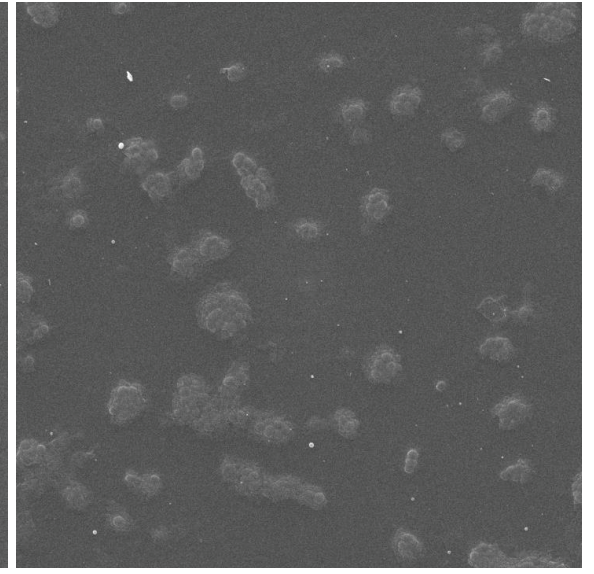

b

Fig. 2. SEM image Primary biofilm formation in Enterococcus faecalis isolated from children of young age: $a$-adhesion; $b$ - co-aggregation stages 
The monolayer formation by isolates in the form of compacted areas with accumulation of cells and microcolonies developing of different sizes, united by the intercellular matrix of cells took place.

Determination of bacterial colonizing ability with the formation of a secondary biofilms found that the longest stage was the stage of re-adsorption in Staphylococcus epidermidis and Enterococcus faecalis that turned to 12 hours (Fig. 3, a), and the shortest was stage of re-aggregation (Fig. 3, b), equal to 2 hours in all detected pathogens.

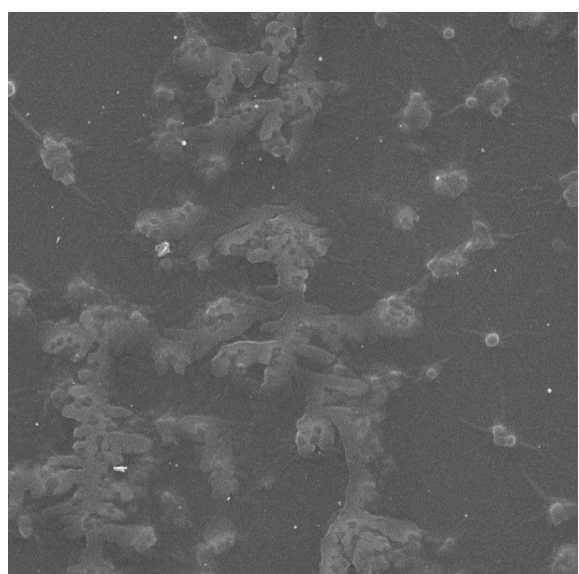

$a$

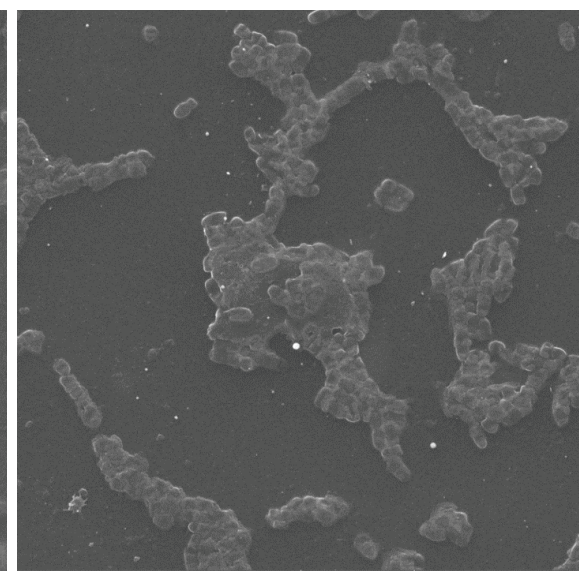

$b$

Fig. 3. SEM image Formation of a secondary biofilms by Enterococcu sfaecalis in young children: $a$-re-adsorption; $b$ - re-aggregation stages

In middle-aged children, among the leading pathogens of pyelonephritis on congenital hydronephrosis background were identified Pseudomonas aeruginosa, Proteus vulgaris, Proteus mirabilis, Klebsiella pneumoniae, Escherichia coli, Enterococcus faecalis, Staphylococcus epidermidis, Candida albicans. And it was established that the duration of adhesion stage was reduced by 1-2 hours compared with it in young children. The duration of adhesion stage for Candida albicans isolates was 2-6 hours.

Due to the formation of intercellular connections, immobilization of planktonic cells and secondary microcolonies formation associated with the primary took place together with the formation of microcavities in matrix, which synthesis undergone due to extracellular substances release in the form of polymer networks that provided biofilms mechanical stability.

It was further found that the finishing in biofilms formation of Escherichia coli occurred in 4-6 hours earlier than it was in young children. The same tendency was observed in isolates of Enterococcus faecalis (in 12 hours), and in Staphylococcus epidermidis (in 8 hours). This fact appeared as a feature of the final stage of biofilms formation by causative agents of pyelonephritis in children with congenital hydronephrosis.

The longest stage in secondary biofilms formation, the stage of re-aggregation in Candida albicans isolates was 14 hours (Fig. 4). In elder children with pyelonephritis on congenital hydronephrosis background, the entire cycle of formation of primary and secondary biofilms by gram-negative bacteria lasted for 26-32 hours, and in gram-positive it was 24-34 hours. The duration of each stage was reduced versus children of younger age categories. The slowest stage was the final stage of secondary biofilms formation, which lasts 4 hours in gram-positive bacteria and 6 hours in gram-negative bacteria, with the exception of Klebsiella pneumoniae isolates, in which the secondary biofilms formation stage lasted for 8 hours (Fig. 5).

A re-merging of microcolonies with the formation of clusters with pores and channels along the lines of formation, surrounded by membrane structures, took place. In the center, the microcolonies were more developed than on the periphery, and between the cells there were long structures like anastomoses and spherical structures that participate in the formation of new microcolonies by colonizing free areas of the substrate. 

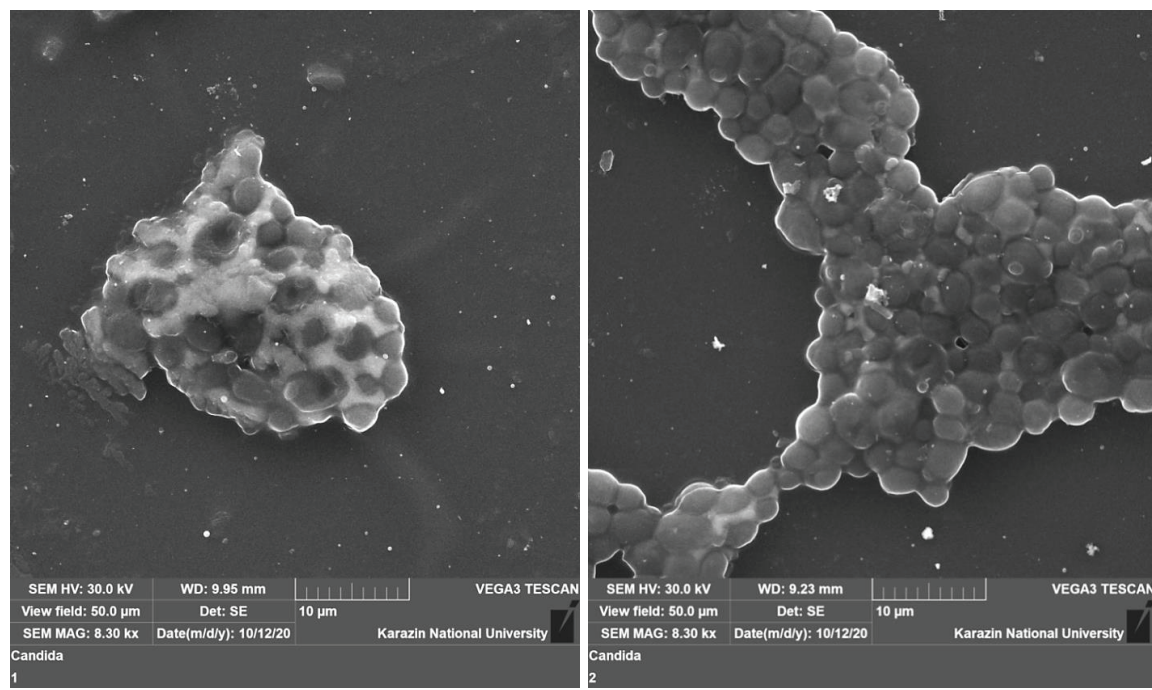

Fig. 4. SEM image Formation of a secondary biofilms in Candida albicans: 1 - start of re-aggregation (after 30 hours from the start of biofilms formation); 2 - re-aggregation after 44 hours



Fig. 5. SEM image Finishing of secondary biofilms formation in Klebsiella pneumonia after 26-34 hours from the start of adhesion

The stage of co-aggregation of isolates in primary biofilms formation was noteworthy: although the beginning of this stage was recorded earlier in 3-4 hours from similar indicators in children of younger age, but the duration of this stage was 10-11 hours and appeared one of the slowest among all stages of primary and secondary biofilms by isolates.

\section{Discussion}

It is known that the formation of biofilms by microorganisms is a complex dynamic process, and consists of attaching cells to the surface with redistribution of cell mass, active cell division with the formation of clusters and exopolymeric mucous matrix. The stage of microbial adhesion to surface undergo due to specific interaction of adhesins or fimbrial lectins of exoplasmic component of bacterial cell with receptors of macroorganism cells [18].The use of scanning electron microscopy revealed that the biofilms of microorganisms that cause pyelonephritis in children with congenital hydronephrosis have not only a complex 3D structural organization, but also after the formation of the primary biofilms isolates actively produce planktonic cells that colonize the body. Then, after 
repeating nonreversible adhesion pathogens begin to grow rapidly and form multicellular layers with the synthesis of a dense exopolymer matrix, which is one of the key moments in the formation of secondary biofilms. Moreover, the cells in the mucous exopolymer matrix are structured in the form of multicellular clusters resembling pillars or trees, has cavities and are divided by channels. The cells of microorganisms in the biofilms have a polymorphic organization: cells with altered morphology were detected, in particular, in Enterococcus faecalis elongated cells were observed. The results obtained in this research do not contradict the leading data in this area $[19,20]$ and supplement it on the stages of biofilms formation by causative agents of pyelonephritis in children.

Study limitations. Exclusion criteria turned to children with associated pathology, children with antibioticotherapy started before collecting specimen (glomerulonephritis, cystitis), and all patients (or their parents) who did not give written informed consent.

Further research deal with searching for antimicrobial agents that can destroy the biofilms formed by pyelonephritis pathogens and the development of methods for rapid diagnosis of the ability of bacteria to form biofilms with subsequent correction (optimization) of antimicrobial therapy in children with pyelonephritis on congenital hydronephrosis background depending on age category.

\section{Conclusions}

1. Scientific data about the stages of biofilms formation by microorganisms, causative agents of pyelonephritis in children were updated and to the classical 5 stages (adhesion, fixation, co-aggregation, clustering, dispersion) the sixth stage (dissemination of planktonic cells formed within the primary biofilms and due to dispersion - separation of the biofilm into fragments with holes), the seventh stage (re-adsorption of planktonic cells on substrates that occurred in 2-3 times faster than in the fixation stage), the eighth stage (re-aggregation, which included the formation not only of a monolayer, but also as a result of grouping of bacterial cells simultaneously with the formation of the biomatrix with the subsequent segmentation of the bacterial secondary biofilms (ninth stage) were added.

2. Adhesion stage of isolates in elder children with pyelonephritis on congenital hydronephrosis background occurred faster in the formation of secondary biofilms than in primary biofilms formation, and it formed the possibility of chronic process and the development of recurrences.

3. The duration of each stage in biofilms formation by causative agents of pyelonephritis in children with congenital hydronephrosis depended on the age of the child and the properties of microorganisms.

\section{Conflict of interests}

The authors declare that they have no conflict of interests.

\section{References}

[1] Gomes, L. C., Mergulhão, F. J. (2017). SEM Analysis of Surface Impact on Biofilm Antibiotic Treatment. Scanning, $2017,1-7$. doi: http://doi.org/10.1155/2017/2960194

[2] Silva-Dias, A., Miranda, I. M., Branco, J., Monteiro-Soares, M., Pina-Vaz, C., Rodrigues, A. G. (2015). Adhesion, biofilm formation, cell surface hydrophobicity, and antifungal planktonic susceptibility: relationship among Candida spp. Frontiers in Microbiology, 6, 205-220. doi: http://doi.org/10.3389/fmicb.2015.00205

[3] Stewart, P. S. (2004). Antibiotic tolerance in biofilms and its role in persistent ifections. 11th International congress on infectious diseases. Cancun, 56.

[4] Gomes, L. C., Silva, L. N., Simões, M., Melo, L. F., Mergulhão, F. J. (2014). Escherichia coliadhesion, biofilm development and antibiotic susceptibility on biomedical materials. Journal of Biomedical Materials Research Part A, 103 (4), $1414-1423$. doi: http://doi.org/10.1002/jbm.a.35277

[5] Das, T., Sharma, P. K., Busscher, H. J., van der Mei, H. C., Krom, B. P. (2010). Role of Extracellular DNA in Initial Bacterial Adhesion and Surface Aggregation. Applied and Environmental Microbiology, 76 (10), 3405-3408. doi: http://doi.org/10.1128/ aem.03119-09

[6] Sugimoto, S., Okuda, K., Miyakawa, R., Sato, M., Arita-Morioka, K., Chiba, A. et. al. (2016). Imaging of bacterial multicellular behaviour in biofilms in liquid by atmospheric scanning electron microscopy. Scientific Reports, 6 (1). doi: http://doi.org/ $10.1038 /$ srep25889 
[7] Moons, P., Michiels, C. W., Aertsen, A. (2009). Bacterial interactions in biofilms. Critical Reviews in Microbiology, 35 (3), 157-168. doi: http://doi.org/10.1080/10408410902809431

[8] Qin, Z., Yang, X., Yang, L., Jiang, J., Ou, Y., Molin, S., Qu, D. (2007). Formation and properties of in vitro biofilms of ica-negative Staphylococcus epidermidis clinical isolates. Journal of Medical Microbiology, 56 (1), 83-93. doi: http://doi.org/10.1099/ jmm.0.46799-0

[9] Moreira, J. M. R., Simões, M., Melo, L. F., Mergulhão, F. J. (2014). Escherichia coli adhesion to surfaces - a thermodynamic assessment. Colloid and Polymer Science, 293 (1), 177-185. doi: http://doi.org/10.1007/s00396-014-3390-x

[10] Hrubanova, K., Krzyzanek, V., Nebesarova, J., Ruzicka, F., Pilat, Z., Samek, O. (2018). Monitoring Candida parapsilosis and Staphylococcus epidermidis Biofilms by a Combination of Scanning Electron Microscopy and Raman Spectroscopy. Sensors, 18 (12), 4089. doi: http://doi.org/10.3390/s18124089

[11] Smith, K., Perez, A., Ramage, G., Lappin, D., Gemmell, C. G., Lang, S. (2008). Biofilm formation by Scottish clinical isolates of Staphylococcus aureus. Journal of Medical Microbiology, 57 (8), 1018-1023. doi: http://doi.org/10.1099/jmm.0.2008/000968-0

[12] Gostev, V. V., Sidorenko, S. V. (2010). Bacterial biofilms and infections. Journal Infectology, 2 (3), 4-15.

[13] Bilko, I. P. (2001). Requirements for the collecting and transport of material for microbiological investigation. Suchasni infectsii, 3, 106-109.

[14] Methodical instructions on application of the unified microbiological (bacteriological) methods of research in clinical diagnostic laboratories (1985). Appendix I to the Order of the Ministry of Health No. 535, 123.

[15] O’Toole, G., Kaplan, H. B., Kolter, R. (2000). Biofilm Formation as Microbial Development. Annual Review of Microbiology, 54 (1), 49-79. doi: http://doi.org/10.1146/annurev.micro.54.1.49

[16] Hobley, L., Harkins, C., MacPhee, C. E., Stanley-Wall, N. R. (2015). Giving structure to the biofilm matrix: an overview of individual strategies and emerging common themes. FEMS Microbiology Reviews, 39 (5), 649-669. doi: http://doi.org/10.1093/ femsre/fuv015

[17] Liu, W., Røder, H. L., Madsen, J. S., Bjarnsholt, T., Sørensen, S. J., Burmølle, M. (2016). Interspecific Bacterial Interactions are Reflected in Multispecies Biofilm Spatial Organization. Frontiers in Microbiology, 7. doi: http://doi.org/10.3389/ fmicb.2016.01366

[18] Pace, J. L. (2006). Biofilms, infection, and antimicrobial therapy. Boca Raton: Taylor \& Francis Group, 495.

[19] Bjarnsholt, T. (2010). Interference of Pseudomonas aeruginosa signalling and biofilm formation for infection control. Expert Reviews in Molecular Medicine, 12. doi: http://doi.org/10.1017/s1462399410001420

[20] Costerton, J. W., Cheng, K. J., Geesey, G. G., Ladd, T. I., Nickel, J. C., Dasgupta, M., Marrie, T. J. (1987). Bacterial Biofilms in Nature and Disease. Annual Review of Microbiology, 41 (1), 435-464. doi: http://doi.org/10.1146/annurev.mi.41.100187.002251 